\title{
ON THE EXISTENCE OF A NON-ZERO LOWER BOUND FOR THE NUMBER OF GOLDBACH PARTITIONS OF AN EVEN INTEGER
}

\author{
SIMON DAVIS
}

\author{
Received 15 March 2002
}

\begin{abstract}
The Goldbach partitions of an even number, given by the sums of two prime addends, form the nonempty set for all integers $2 n$ with $2 \leq n \leq 2 \times 10^{14}$. It will be shown how to determine by the method of induction the existence of a non-zero lower bound for the number of Goldbach partitions of all even integers greater than or equal to 4 . The proof depends on contour arguments for complex functions in the unit disk.
\end{abstract}

2000 Mathematics Subject Classification: 11L03, 11L20, 11P32, 11P83.

The order estimates of the number of partitions of an even integer into a sum of primes typically have an error term. However, when the partitions are restricted to the set of sums of two primes, the form of the error term implies that the estimate does not exclude the possibility of zero being obtained. If the constant $c$ in the error term is specified, then the integer typically should be greater than some exponential function of $c$ for the lower bound to be non-zero.

For example, an estimate of the number of partitions into the sum of two primes has been obtained by using an integral representation of the density of prime powers in a given interval [5]:

$$
G(2 s)=\int_{2}^{2 s-2} \frac{d y}{\log y \cdot \log (2 s-y)}+O\left(\frac{2 s}{\log ^{3} 2 s}\right)+O\left(\frac{2 s}{\log ^{q} 2 s}\right) .
$$

A non-zero lower bound for $G(2 s)$ only exists if

$$
\begin{aligned}
\frac{2 s-4}{\log ^{2} 2 s} & >c \cdot \frac{2 s}{\log ^{3} 2 s} \frac{1}{(1+\log 2 / \log s)^{3}}, \\
s & >\frac{1}{2} e^{2 c+c^{\prime} / c^{q-3}}, \quad c^{\prime} \text { constant. }
\end{aligned}
$$

This problem does not arise in the order estimates in the lower bound for the number of partitions of odd integers into the sum of three primes because the error term is typically less than the leading estimate by a factor of $1 /(\log N)^{A}$, where $A$ can be chosen to be arbitrarily large [6]. 
It is conventional to obtain an estimate of the number of partitions of an integer into the sums of primes by considering the following exponential sums [2, 4]:

$$
R(\alpha)=\sum_{p} e^{2 \pi i p \alpha}, \quad S(\alpha)=\sum_{p}(\log p) e^{2 \pi i n \alpha},
$$

and the functions

$$
g(\alpha)=R(\alpha)^{2} e^{-2 \pi i n \alpha}, \quad h(\alpha)=S(\alpha)^{2} e^{-2 \pi i n \alpha},
$$

with the integrals

$$
\begin{aligned}
& G(n)=\int_{0}^{1} R(\alpha)^{2} e^{-2 \pi i n \alpha} d \alpha=\sum_{p_{1}, p_{2}} \int_{0}^{1} e^{2 \pi i\left(p_{1}+p_{2}-n\right) \alpha} d \alpha \\
& H(n)=\int_{0}^{1} S(\alpha)^{2} e^{-2 \pi i n \alpha} d \alpha=\sum_{p_{1}, p_{2}}\left(\log p_{1}\right)\left(\log p_{2}\right) \int_{0}^{1} e^{2 \pi i\left(p_{1}+p_{2}-n\right) \alpha} d \alpha .
\end{aligned}
$$

Since the integral vanishes when $p_{1}+p_{2} \neq n, G(n)$ equals the number of Goldbach partitions of $n$ with the order of summands relevant, and the two functions $G(n)$ and $H(n)$ are simultaneously greater than zero. The method of induction will be used to determine the existence of a non-zero lower bound for the number of Goldbach partitions of an even integer.

Suppose that the even integer $n-2$ can be written as the sum of two prime numbers. Then

$$
H(n-2)=\frac{1}{2 \pi} \int_{0}^{2 \pi} S(\theta)^{2} e^{-i(n-2) \theta} d \theta>0, \quad \theta=2 \pi \alpha .
$$

This integral can be viewed as a contour integral over the unit circle

$$
\frac{1}{2 \pi} \oint \vec{v} \cdot \overrightarrow{d l}
$$

where

$$
\vec{v}^{(n-2)}=\left(v_{r}^{(n-2)}, v_{\theta}^{(n-2)}\right), \quad \overrightarrow{d l}=(0, d \theta) .
$$

Let $\vec{v}^{(n)}(r=1)=e^{-2 i \theta} \vec{v}^{(n-2)}(r=1)$. The extrapolation of the function into the disk is either $z^{-2}(r, \theta) \vec{v}^{(n-2)}(r, \theta)$ or $\bar{z}^{2} \vec{v}^{(n-2)}(r, \theta)$, after excluding fractional powers of $z$, $\bar{z}$ and independence of the coefficient of $\vec{v}^{(m-2)}$ with respect to the radial coordinate. By Stokes' theorem, the nonvanishing of the line integral implies that $\vec{v}^{(n-2)}(r, \theta) \neq \vec{\nabla} \chi$, where $\chi$ is a nonsingular function of $r, \theta$.

Consider $z^{-2} \vec{\nabla} \chi=r^{-2} e^{-2 i \theta} \vec{\nabla} \chi(r, \theta)$ and suppose that there is a singularity in $\chi$ at $\theta=\theta_{s}$. If $\vec{\nabla} \psi_{1}(r, \theta)=z^{-2} \vec{\nabla} \chi(r, \theta)$, then

$$
\psi_{1}\left(r, \theta_{s}\right)=\left.e^{-2 i \theta_{s}} \int d r r^{-2} \frac{\partial \chi(r, \theta)}{\partial r}\right|_{\theta_{s}}
$$


so that when $\chi\left(r, \theta_{s}\right)$ is a singular function of the radial variable $r, \psi_{1}\left(r, \theta_{s}\right)$ also will be a singular function of $r$, giving rise to a non-zero surface integral

$$
\begin{gathered}
\iint_{D} \vec{\nabla} \times \vec{v}^{(n)}(r, \theta) \cdot d \vec{S}, \\
H(n)=\frac{1}{2 \pi} \int_{0}^{2 \pi} S(\theta)^{2} e^{-i n \theta} d \theta=\frac{1}{2 \pi} \oint \vec{v}^{(n)} \cdot \overrightarrow{d l} \neq 0 .
\end{gathered}
$$

Next consider the function defined by $\vec{\nabla} \psi_{2}(r, \theta)=\bar{z}^{2} \vec{\nabla} \chi(r, \theta)$. Then

$$
\psi_{2}\left(r, \theta_{s}\right)=\left.e^{-2 i \theta_{s}} \int d r r^{2} \frac{\partial \chi(r, \theta)}{\partial r}\right|_{\theta_{s}}
$$

and the singularity in $\chi(r, \theta)$ may be removed by the integral. However, by Stokes' theorem,

$$
\oint_{C} f(z, \bar{z}) d z=\iint_{D} \frac{\partial f(z, \bar{z})}{\partial \bar{z}} d \bar{z} \wedge d z
$$

If $f(z, \bar{z})=-(i / r) e^{-i \theta}\left(\partial \psi_{2}(z, \bar{z}) / \partial \theta\right)$, the surface integral receives a contribution from the integral

$$
-\frac{i}{\pi} \iint_{D} \bar{z} \frac{e^{-i \theta}}{r} \frac{\partial \chi(r, \theta)}{\partial \theta} d \bar{z} \wedge d z
$$

Combining the two integrals gives

$$
-\frac{i}{\pi} \iint_{D} \bar{z} \frac{e^{-i \theta}}{r} \frac{\partial \chi(r, \theta)}{\partial \theta} d \bar{z} \wedge d z-\frac{i}{2 \pi} \iint_{D} \bar{z}^{2} \frac{\partial}{\partial \bar{z}}\left(\frac{e^{-i \theta}}{r} \frac{\partial \chi(r, \theta)}{\partial \theta}\right) d \bar{z} \wedge d z,
$$

and using

$$
\frac{\partial}{\partial \bar{z}}=\frac{1}{2} e^{2 i \theta} \frac{\partial}{\partial r}+\frac{i}{2} \frac{e^{i \theta}}{r} \frac{\partial}{\partial \theta}
$$

gives rise to the following expression:

$$
\begin{aligned}
\frac{2}{\pi} \iint_{D} r e^{-2 i \theta} \frac{\partial \chi(r, \theta)}{\partial \theta} d r d \theta & +\frac{1}{2 \pi} \iint_{D} r^{2} e^{-2 i \theta} \frac{\partial^{2} \chi(r, \theta)}{\partial \theta \partial r} d r d \theta \\
& +\frac{i}{2 \pi} \iint_{D} r e^{-2 i \theta} \frac{\partial^{2} \chi(r, \theta)}{\partial \theta^{2}} d r d \theta
\end{aligned}
$$

Recalling that

$$
\begin{aligned}
v_{\theta}^{(n-2)}(r=1) & =S(\theta)^{2} e^{-i(n-2) \theta} \\
& =\sum_{p_{1}, p_{2}}\left(\log p_{1}\right)\left(\log p_{2}\right) e^{i\left(p_{1}+p_{2}-(n-2)\right) \theta}=\left.\frac{1}{r} \frac{\partial \chi}{\partial \theta}\right|_{r=1},
\end{aligned}
$$


it follows that

$$
\begin{aligned}
\chi(r=1, \theta)= & \sum_{\substack{p_{1}, p_{2} \\
p_{1}+p_{2} \neq n-2}} \frac{\left(\log p_{1}\right)\left(\log p_{2}\right)}{i\left(p_{1}+p_{2}-(n-2)\right)} e^{i\left(p_{1}+p_{2}-(n-2)\right) \theta} \\
& +\sum_{p_{1}+p_{2}=n-2}\left(\log p_{1}\right)\left(\log p_{2}\right) \theta
\end{aligned}
$$

If this function on the unit circle is extended to the entire disk, using the variables $z$, $\bar{z}$, it is

$$
\begin{aligned}
\chi(z, \bar{z})= & i \sum_{p_{1}+p_{2}<n-2} \frac{\left(\log p_{1}\right)\left(\log p_{2}\right)}{(n-2)-\left(p_{1}+p_{2}\right)} \bar{z}^{\left((n-2)-\left(p_{1}+p_{2}\right)\right)} \\
& -\frac{i}{2} \sum_{p_{1}+p_{2}=n-2}\left(\log p_{1}\right)\left(\log p_{2}\right) \ln \left(\frac{z}{\bar{z}}\right) \\
& -i \sum_{p_{1}+p_{2}>n-2} \frac{\left(\log p_{1}\right)\left(\log p_{2}\right)}{p_{1}+p_{2}-(n-2)} z^{p_{1}+p_{2}-(n-2)} .
\end{aligned}
$$

This function is nonsingular, and except for the second term, it is well defined everywhere throughout the disk besides the origin. Its value at the origin is undefined as it depends on the direction in which the limit $r \rightarrow 0$ is taken. However, the gradient would be well defined, and furthermore, it is nonsingular. Setting $\vec{v}^{(n-2)}$ equal to a nonsingular gradient is not consistent with a nonvanishing contour integral $\oint_{C} \vec{v}^{(n-2)} \cdot \overrightarrow{d l}$.

However, if this form is chosen for $\chi(z, \bar{z})$, the expression for $\oint_{C} \vec{v}^{(n)} \cdot \overrightarrow{d l}$ based on the function $\psi_{2}(r, \theta)$ can be computed. In terms of $r, \theta$,

$$
\begin{aligned}
\chi(r, \theta)= & i \sum_{\substack{p_{1}, p_{2} \\
p_{1}+p_{2}<n-2}} \frac{\left(\log p_{1}\right)\left(\log p_{2}\right)}{(n-2)-\left(p_{1}+p_{2}\right)} r^{(n-2)-\left(p_{1}+p_{2}\right)} e^{-i\left((n-2)-\left(p_{1}+p_{2}\right)\right) \theta} \\
& +\sum_{p_{1}+p_{2}=n-2}\left(\log p_{1}\right)\left(\log p_{2}\right) \theta \\
& -i \sum_{\substack{p_{1}, p_{2} \\
p_{1}+p_{2}>n-2}} \frac{\left(\log p_{1}\right)\left(\log p_{2}\right)}{p_{1}+p_{2}-(n-2)} r^{p_{1}+p_{2}-(n-2)} e^{i\left(p_{1}+p_{2}-(n-2)\right) \theta} \\
4 \frac{\partial \chi(r, \theta)}{\partial \theta}+ & r \frac{\partial^{2} \chi(r, \theta)}{\partial r \partial \theta}+i \frac{\partial^{2} \chi(r, \theta)}{\partial \theta^{2}} \\
= & 4 \sum_{p_{1}+p_{2} \neq 2} \frac{\left(\log p_{1}\right)\left(\log p_{2}\right)}{p_{1}+p_{2}-(n-2)} r^{\left|p_{1}+p_{2}-(n-2)\right|} e^{i\left(p_{1}+p_{2}-(n-2)\right) \theta} \\
& +\sum_{p_{1}+p_{2}=n-2}\left(\log p_{1}\right)\left(\log p_{2}\right)
\end{aligned}
$$


since $\int_{0}^{2 \pi} e^{i\left(p_{1}+p_{2}-n\right) \theta} d \theta=0$ when $p_{1}+p_{2} \neq n$, and the integral becomes

$$
\begin{array}{r}
\frac{2}{\pi} \sum_{p_{1}+p_{2}=n}\left(\log p_{1}\right)\left(\log p_{2}\right) \int_{0}^{1} r^{3} d r \cdot \int_{0}^{2 \pi} d \theta \\
=\sum_{p_{1}+p_{2}=n}\left(\log p_{1}\right)\left(\log p_{2}\right)=H(n) .
\end{array}
$$

If instead the sum $R(\theta)$ is used, the result is $\sum_{p_{1}+p_{2}=n} 1=G(n)$.

The integral expression in $(z, \bar{z})$ coordinates is

$$
\begin{gathered}
-\frac{i}{\pi} \iint_{D}\left[\sum_{p_{1}+p_{2}=n-2} \frac{\left(\log p_{1}\right)\left(\log p_{2}\right)}{z}+\sum_{p_{1}+p_{2}>n-2}\left(\log p_{1}\right)\left(\log p_{2}\right) z^{p_{1}+p_{2}-(n-1)}\right. \\
\left.+\frac{1}{z} \sum_{p_{1}+p_{2}<n-2}\left(\log p_{1}\right)\left(\log p_{2}\right) \bar{z}^{(n-2)-\left(p_{1}+p_{2}\right)}\right] \bar{z} d \bar{z} \wedge d z .
\end{gathered}
$$

By the complex Green formula, it follows that each of the integrals except

$$
-\frac{i}{\pi} \iint_{D} \sum_{p_{1}+p_{2}>n-2} z^{p_{1}+p_{2}-(n-1)} \bar{z} d \bar{z} \wedge d z
$$

vanishes. Using the Cauchy formula for nonanalytic functions $f(z, \bar{z})[1]$

$$
f\left(z_{0}\right)=\frac{1}{2 \pi i} \int_{C} \frac{f(z)}{z-z_{0}} d z+\frac{1}{2 \pi i} \iint_{D} \frac{\partial f / \partial \bar{z}}{z-z_{0}} d z \wedge d \bar{z}
$$

and the vanishing of $\bar{z} z^{p_{1}+p_{2}-(n-1)}$ for $p_{1}+p_{2}>n-2$ at the origin, it follows that

$$
\begin{aligned}
-\frac{i}{\pi} \iint_{D} \bar{z} z^{p_{1}+p_{2}-(n-1)} d \bar{z} \wedge d z & =\frac{i}{\pi} \iint_{D} \bar{z} \frac{z^{p_{1}+p_{2}-(n-2)}}{z} d z \wedge d \bar{z} \\
& =-\frac{i}{\pi} \int_{C} \frac{\bar{z}^{2}}{2} \frac{z^{p_{1}+p_{2}-(n-2)}}{z} d z \\
& =-\frac{i}{2 \pi} \int_{0}^{2 \pi} e^{i\left(p_{1}+p_{2}-(n-1)\right) \theta} e^{-2 i \theta} \cdot i e^{i \theta} d \theta \\
& =-\frac{i}{2 \pi} \int_{0}^{2 \pi} i e^{i\left(p_{1}+p_{2}-n\right) \theta} d \theta=\delta_{p_{1}+p_{2}, n}
\end{aligned}
$$

so that the sum above is

$$
\sum_{p_{1}+p_{2}>n-2}\left(\log p_{1}\right)\left(\log p_{2}\right) \delta_{p_{1}+p_{2}, n}=\sum_{p_{1}+p_{2}=n}\left(\log p_{1}\right)\left(\log p_{2}\right)=H(n) .
$$

This result confirms the equivalence of the integral expression in terms of $r, \theta$ and $z, \bar{z}$. 
Now consider the extrapolation of $\chi(r=1, \theta)$ based on function

$$
\begin{aligned}
\chi(z, \bar{z})= & i \sum_{p_{1}+p_{2}<n-2} \frac{\left(\log p_{1}\right)\left(\log p_{2}\right)}{(n-2)-\left(p_{1}+p_{2}\right)} z^{-\left((n-2)-\left(p_{1}+p_{2}\right)\right)} \\
& -\frac{i}{2} \sum_{p_{1}+p_{2}=n-2}\left(\log p_{1}\right)\left(\log p_{2}\right) \ln \left(\frac{z}{\bar{z}}\right) \\
& -i \sum_{p_{1}+p_{2}>n-2} \frac{\left(\log p_{1}\right)\left(\log p_{2}\right)}{\left(p_{1}+p_{2}\right)-(n-2)} z^{p_{1}+p_{2}-(n-2)}
\end{aligned}
$$

In terms of $r, \theta$, this expression becomes

$$
\begin{aligned}
x(r, \theta)= & i \sum_{p_{1}+p_{2}<n-2} \frac{\left(\log p_{1}\right)\left(\log p_{2}\right)}{(n-2)-\left(p_{1}+p_{2}\right)} r^{-\left((n-2)-\left(p_{1}+p_{2}\right)\right)} e^{-i\left((n-2)-\left(p_{1}+p_{2}\right)\right) \theta} \\
& +\sum_{p_{1}+p_{2}=n-2}\left(\log p_{1}\right)\left(\log p_{2}\right) \theta \\
& -i \sum_{p_{1}+p_{2}>n-2} \frac{\left(\log p_{1}\right)\left(\log p_{2}\right)}{p_{1}+p_{2}-(n-2)} r^{p_{1}+p_{2}-(n-2)} e^{i\left(p_{1}+p_{2}-(n-2)\right) \theta}, \\
4 \frac{\partial \chi}{\partial \theta}+ & r \frac{\partial^{2} \chi}{\partial \theta \partial r}+i \frac{\partial^{2} \chi}{\partial \theta^{2}}\left(\log p_{1}\right)\left(\log p_{2}\right) r^{-\left((n-2)-\left(p_{1}+p_{2}\right)\right)} e^{-i\left((n-2)-\left(p_{1}+p_{2}\right)\right) \theta} \\
= & 4 \sum_{p_{1}+p_{2}<n-2}\left(\log p_{1}\right)\left(\log p_{2}\right) \\
& +4 \sum_{p_{1}+p_{2}=n-2}\left(\log p_{1}\right)\left(\log p_{2}\right) r^{p_{1}+p_{2}-(n-2)} e^{i\left(p_{1}+p_{2}-(n-2)\right) \theta} \\
& +4 \sum_{p_{1}+p_{2}>n-2}\left(\log p_{1}\right)\left(\log p_{2}\right)\left(p_{1}+p_{2}-(n-2)\right) \\
& +\sum_{p_{1}+p_{2}<n-2} \times r^{-\left((n-2)-\left(p_{1}+p_{2}\right)\right)} e^{-i\left((n-2)-\left(p_{1}+p_{2}\right)\right) \theta} \\
& +\sum_{p_{1}+p_{2}>n-2}\left(\log p_{1}\right)\left(\log p_{2}\right)\left(p_{1}+p_{2}-(n-2)\right) r^{p_{1}+p_{2}-(n-2)} e^{i\left(p_{1}+p_{2}-(n-2)\right) \theta} \\
& +\sum_{p_{1}+p_{2}<n-2}\left(\log p_{1}\right)\left(\log p_{2}\right)\left((n-2)-\left(p_{1}+p_{2}\right)\right) \\
& +\sum_{n-2}\left(\log p_{1}\right)\left(\log p_{2}\right)\left(p_{1}+p_{2}-(n-2)\right) r^{p_{1}+p_{2}-(n-2)} e^{i\left(p_{1}+p_{2}-(n-2)\right) \theta}
\end{aligned}
$$

The integral

$$
\frac{1}{2 \pi} \iint_{D} e^{-2 i \theta}\left(4 \frac{\partial \chi}{\partial \theta}+r \frac{\partial^{2} \chi}{\partial \theta \partial r}+i \frac{\partial^{2} \chi}{\partial \theta^{2}}\right) r d r d \theta
$$


equals

$$
\begin{aligned}
\frac{2}{\pi} \sum_{p_{1}+p_{2}>n-2}\left(\log p_{1}\right)\left(\log p_{2}\right) \int_{0}^{2 \pi} e^{i\left(p_{1}+p_{2}-n\right) \theta} d \theta \int_{0}^{1} r^{p_{1}+p_{2}-(n-3)} d r \\
+\frac{1}{2 \pi} \sum_{p_{1}+p_{2}>n-2}\left(\log p_{1}\right)\left(\log p_{2}\right) \cdot\left(p_{1}+p_{2}-(n-2)\right) \\
\quad \cdot \int_{0}^{2 \pi} e^{i\left(p_{1}+p_{2}-(n-2)\right) \theta} e^{-2 i \theta} d \theta \cdot \int_{0}^{1} r^{p_{1}+p_{2}-(n-3)} d r \\
-\frac{1}{2 \pi} \sum_{p_{1}+p_{2}>n-2}\left(\log p_{1}\right)\left(\log p_{2}\right) \\
\cdot\left(p_{1}+p_{2}-(n-2)\right) \cdot \int_{0}^{2 \pi} e^{i\left(p_{1}+p_{2}-(n-2)\right) \theta} \cdot e^{-2 i \theta} d \theta \int_{0}^{1} r^{p_{1}+p_{2}-(n-3)} d r .
\end{aligned}
$$

When $p_{1}+p_{2}=n$, this sum equals

$$
\begin{gathered}
\frac{2}{\pi} \sum_{p_{1}+p_{2}=n}\left(\log p_{1}\right)\left(\log p_{2}\right) \cdot \frac{1}{4} \cdot 2 \pi+\frac{1}{2 \pi} \cdot 2 \cdot 2 \pi \cdot \frac{1}{4} \sum_{p_{1}+p_{2}=n}\left(\log p_{1}\right)\left(\log p_{2}\right) \\
-\frac{1}{2 \pi} \cdot 2 \cdot 2 \pi \cdot \frac{1}{4} \sum_{p_{1}+p_{2}=n}\left(\log p_{1}\right)\left(\log p_{2}\right)=H(n) .
\end{gathered}
$$

Now consider the integral in terms of the coordinates $z, \bar{z}$. Since

$$
\begin{aligned}
\frac{\partial x}{\partial \bar{z}}= & \frac{i}{2} \sum_{p_{1}+p_{2}=n-2} \frac{\left(\log p_{1}\right)\left(\log p_{2}\right)}{\bar{z}} \\
\frac{\partial x}{\partial z}= & -i \sum_{p_{1}+p_{2}<n-2}\left(\log p_{1}\right)\left(\log p_{2}\right) z^{-\left((n-1)-\left(p_{1}+p_{2}\right)\right)} \\
& -\frac{i}{2} \sum_{p_{1}+p_{2}=n-2} \frac{\left(\log p_{1}\right)\left(\log p_{2}\right)}{z} \\
& -i \sum_{p_{1}+p_{2}>n-2}\left(\log p_{1}\right)\left(\log p_{2}\right) z^{p_{1}+p_{2}-(n-1)}
\end{aligned}
$$

the following identities are obtained:

$$
\begin{aligned}
e^{-2 i \theta} \frac{\partial \chi(r, \theta)}{\partial \theta}= & i \bar{z} \frac{\partial \chi}{\partial z}-i \frac{\bar{z}^{2}}{z} \frac{\partial \chi}{\partial \bar{z}} \\
= & \sum_{p_{1}+p_{2}<n-2}\left(\log p_{1}\right)\left(\log p_{2}\right) \bar{z} z^{-\left((n-1)-\left(p_{1}+p_{2}\right)\right)} \\
& +\sum_{p_{1}+p_{2}=n-2}\left(\log p_{1}\right)\left(\log p_{2}\right) \frac{\bar{z}}{z} \\
& +\sum_{p_{1}+p_{2}>n-2}\left(\log p_{1}\right)\left(\log p_{2}\right) \bar{z} z^{p_{1}+p_{2}-(n-1)}
\end{aligned}
$$




$$
\begin{aligned}
\frac{e^{-i \theta}}{r} \frac{\partial \chi}{\partial \theta}= & \sum_{p_{1}+p_{2}<n-2}\left(\log p_{1}\right)\left(\log p_{2}\right) z^{-\left((n-1)-\left(p_{1}+p_{2}\right)\right)} \\
& +\sum_{p_{1}+p_{2}=n-2} \frac{\left(\log p_{1}\right)\left(\log p_{2}\right)}{z} \\
& +\sum_{p_{1}+p_{2}>n-2}\left(\log p_{1}\right)\left(\log p_{2}\right) z^{p_{1}+p_{2}-(n-1)}
\end{aligned}
$$

and $\bar{z}^{2}(\partial / \partial \bar{z})\left(\left(e^{-i \theta} / r\right)(\partial x / \partial \theta)\right)=0$, so that the integral in $(z, \bar{z})$ coordinates is

$$
\begin{aligned}
-\frac{i}{\pi} \iint_{D} & {\left[\sum_{p_{1}+p_{2}<n-2}\left(\log p_{1}\right)\left(\log p_{2}\right) \bar{z} z^{-\left((n-1)-\left(p_{1}+p_{2}\right)\right)}+\sum_{p_{1}+p_{2}=n-2}\left(\log p_{1}\right)\left(\log p_{2}\right) \frac{\bar{z}}{z}\right.} \\
& \left.+\sum_{p_{1}+p_{2}>n-2}\left(\log p_{1}\right)\left(\log p_{2}\right) \bar{z} z^{p_{1}+p_{2}-(n-1)}\right] d \bar{z} \wedge d z .
\end{aligned}
$$

The first term in the expression is the integral of a singular function. Using the identity

$$
\iint_{D} \frac{\partial f / \partial \bar{z}}{\left(z-z_{0}\right)^{k}} d \bar{z} \wedge d z=\int_{C} \frac{f(z, \bar{z})}{\left(z-z_{0}\right)^{k}} d z-\frac{1}{(k-1) !} 2 \pi i \frac{\partial^{(k-1)} f}{\partial z^{(k-1)}}\left(z_{0}\right),
$$

it follows that this term vanishes.

However, this result suggests that there would be a non-zero contribution from the singular terms once an additional positive-definite function is included in the integral. An example of such a function is $e^{\delta(1-\bar{z} z)}=e^{\delta\left(1-r^{2}\right)}$. Multiplication by this factor in the integral over $r$ and $\theta$ gives

$$
\begin{aligned}
& \frac{2}{\pi} \int_{0}^{1} r^{3} e^{\delta\left(1-r^{2}\right)} d r \cdot 2 \pi \sum_{p_{1}+p_{2}=n}\left(\log p_{1}\right)\left(\log p_{2}\right) \\
& \quad=4 I_{3}(\delta) \sum_{p_{1}+p_{2}=n}\left(\log p_{1}\right)\left(\log p_{2}\right)=4 I_{3}(\delta) H(n),
\end{aligned}
$$

where $I_{3}(\delta)$ is the integral over $r$ which equals $1 / 4$ when $\delta=0$.

With a support function in the integral over $z, \bar{z}$ and use of the identity for higher powers of $\left(z-z_{0}\right)^{k}$ in the denominator, linear combinations of $H(m), m \leq n$, are obtained. As $\delta$ is varied, or different support functions are chosen, these linear combinations will change, and given the nonvanishing of $H(m)$ for $m \leq n-2$, equivalence of the integrals in the $r, \theta$ coordinates and the $z, \bar{z}$ coordinates implies a nontrivial result for the magnitude of $H(n)$. The same conclusions hold for $G(n)$.

Furthermore, considering the different extrapolations of $\chi(z, \bar{z})$ to the interior of the disk, it can be seen that the use of the nonsingular expression for $\chi(z, \bar{z})$ does not yield any constraints on $H(n)$ and therefore, it would be consistent to set this value to zero. Conversely, the feasibility of setting $H(n)$ equal to zero implies that $\chi(z, \bar{z})$ must be nonsingular, which would, in turn, imply that $H(n-2)$ vanishes, contrary to the original assumption that it is non-zero. Thus, the contour integral argument provides a method for deducing a non-zero value for $H(n)$, given that $H(m) \neq 0$ for $4 \leq m \leq n-2$. 
By induction, it would follow then that $H(n)$ is non-zero for all values of $n$. The nonvanishing of $H(n)$ is sufficient for a proof of a lower bound for the number of Goldbach partitions of any even number greater than or equal to 4 , since the induction argument also holds for $G(n)=\sum_{p_{1}+p_{2}=n} 1$.

The special role of the primes in the integrals of the summed expression of exponential functions of $\theta$ can be elucidated by noting that any exponential with exponent given by $2 \pi i$ multiplied by a rational number $e^{2 \pi i(m / n) \alpha}, \alpha=1$, can be obtained as a power $e^{(2 \pi i(p / n) \alpha) k}, \alpha=1$, for some $k$ if $p$ is a prime number. Suppose that

$$
e^{2 \pi i(m / n)}=\left(e^{2 \pi i\left(p_{1} / n\right)}\right)^{k_{1}}=\left(e^{2 \pi i\left(p_{2} / n\right)}\right)^{k_{2}}, \quad m<\left[\frac{n}{2}\right],
$$

so that for every even numerator,

$$
e^{2 \pi i(2 m / n)}=e^{2 \pi i\left(\left(p_{1} k_{1}+p_{2} k_{2}\right) / n\right)}
$$

for some $p_{1}, p_{2}$. Since the number of Goldbach partitions is assumed to be positive for $2 m \leq n-2$, there is a pair of primes $p_{1}, p_{2}$ such that $2 m=p_{1}+p_{2}$. Consequently, this exponential can be equated with $e^{2 \pi i\left(\left(p_{1}+p_{2}\right) / n\right)}$, revealing the effect of identification of the exponential of a sum of fractions having prime numerators with an exponential of a fraction having an even numerator less than $n$.

This property can be extended to $n+2$ by noting that

$$
e^{2 \pi i\left(2 m^{\prime} /(n+2)\right)}=e^{2 \pi i\left(p_{1}^{\prime} k_{1}^{\prime}+p_{2}^{\prime} k_{2}^{\prime}\right) /(n+2)}
$$

for any pair of primes $p_{1}^{\prime}, p_{2}^{\prime}$.

It has been shown that for any even integer $2 m \leq n-2$, there exist integers $k_{1}$, $k_{2}$ such that $p_{1} k_{1}+p_{2} k_{2} \equiv 2 m(\bmod n)$ and $p_{1}\left(k_{1}-1\right)+p_{2}\left(k_{2}-1\right) \equiv 0(\bmod n)$. This property can be extended to a congruence modulo $n+2$.

Since the number of incongruent solutions to the equations

$$
a_{1} x_{1}+\cdots+a_{\ell} x_{\ell}+b \equiv 0(\bmod n),
$$

where $\operatorname{gcd}\left(a_{1}, \ldots, a_{\ell}, n\right) \mid b$, is $n^{\ell-1} \operatorname{gcd}\left(a_{1}, \ldots, a_{\ell}, n\right)$ [3], the number of solutions to the congruence $p_{1} k_{1}+p_{2} k_{2} \equiv 2 m(\bmod n)$ for a pair of primes $p_{1}, p_{2}$ is $n$. Conversely, the number of solutions to $a_{1} k_{1}+a_{2} k_{2} \equiv 2 m(\bmod n)$ for fixed $k_{1}, k_{2}$ is equal to $n \operatorname{gcd}\left(k_{1}, k_{2}, n\right)$, and by assumption, the intersection of this set with the solutions to $a_{1}\left(k_{1}-1\right)+a_{2}\left(k_{2}-1\right) \equiv 0(\bmod n)$ includes the prime pair $\left(p_{1}, p_{2}\right)$. For fixed $k_{1}^{\prime}, k_{2}^{\prime}$, the properties of the intersection of solutions sets of $a_{1}^{\prime} k_{1}^{\prime}+a_{2}^{\prime} k_{2}^{\prime} \equiv 2 m^{\prime}(\bmod n+2)$ and $a_{1}^{\prime}\left(k_{1}^{\prime}-1\right)+a_{2}^{\prime}\left(k_{2}^{\prime}-1\right) \equiv 0(\bmod n+2)$ will be determined by the linearity of the congruence relations. Extending the solution set $D_{n}$ to $D_{n+2}$, the existence of a prime pair $\left(p_{1}^{\prime}, p_{2}^{\prime}\right)$ in the set $D_{n+2}$ can be deduced from the density of prime pairs in the planar domain represented by the congruence relation modulo $n+2$.

It follows that the exponentials with exponents containing fractions with prime numerators can be used as an appropriate basis for all exponentials of the type $e^{2 \pi i(\mathrm{~m} / \mathrm{n})}$. This basis can be extrapolated to represent $e^{2 \pi i \alpha}, \alpha \neq 1$, by means of the functional analytic technique of extending the domain of functions from the set of rational numbers forming a dense subset of the continuous interval $[0,1]$ to the entire interval. 
ACKNOWLEDGMENT. This work has been supported by a Research Fellowship from the Alexander von Humboldt Foundation.

\section{REFERENCES}

[1] S. R. Bell, The Cauchy Transform, Potential Theory, and Conformal Mapping, Studies in Advanced Mathematics, CRC Press, Boca Raton, Florida, 1992.

[2] G. H. Hardy and J. E. Littlewood, Some problems of "partitio numerorum"; (V): A further contribution to the study of Goldbach's problem, Proc. London Math. Soc. (2) 22 (1924), 46-56.

[3] L. K. Hua, Introduction to Number Theory, Springer-Verlag, Berlin, 1982.

[4] M. B. Nathanson, Additive Number Theory. The Classical Bases, Graduate Texts in Mathematics, vol. 164, Springer-Verlag, New York, 1996.

[5] A. Peretti, The Goldbach problem, Bull. Number Theory Related Topics 10 (1986), no. 2, 32-47.

[6] I. M. Vinogradov, The Method of Trigonometrical Sums in the Theory of Numbers, Interscience Publishers, New York, 1954.

Simon Davis: Institut für Mathematik, Universität Potsdam, D-14415 Potsdam, Germany

E-mail address: davis@math.uni-potsdam.de 


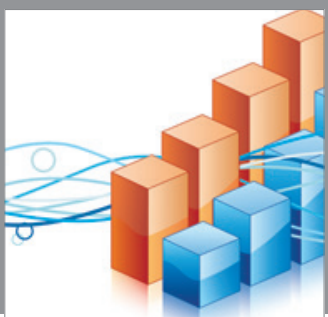

Advances in

Operations Research

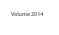

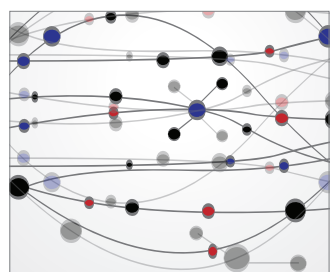

\section{The Scientific} World Journal
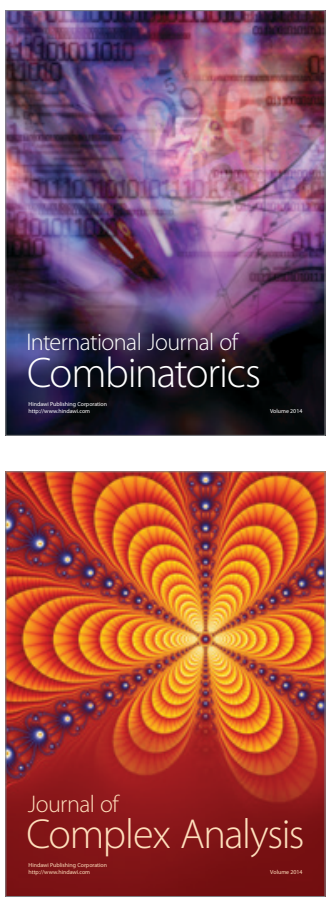

International Journal of

Mathematics and

Mathematical

Sciences
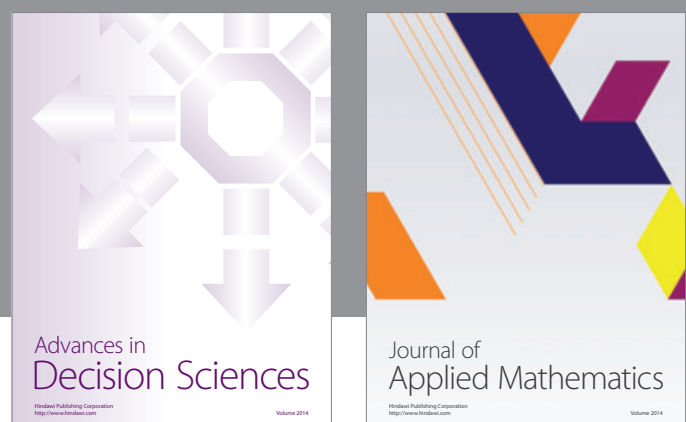

Journal of

Applied Mathematics
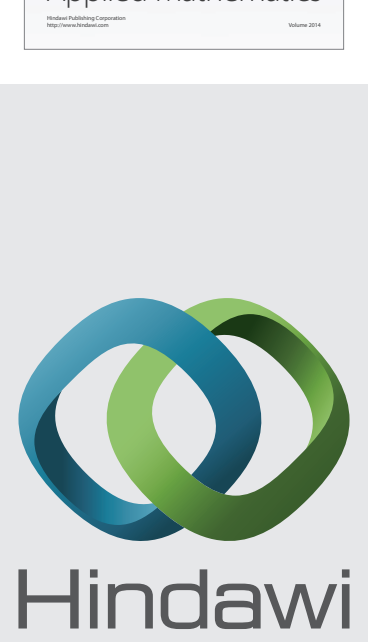

Submit your manuscripts at http://www.hindawi.com
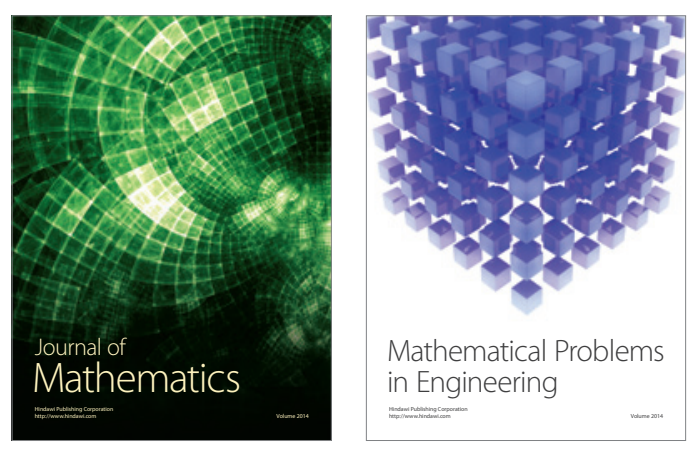

Mathematical Problems in Engineering
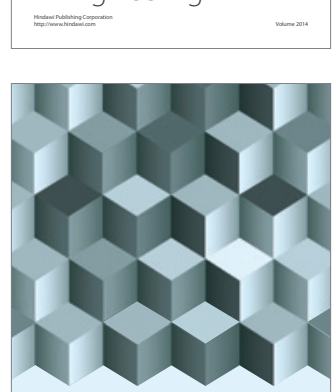

Journal of

Function Spaces
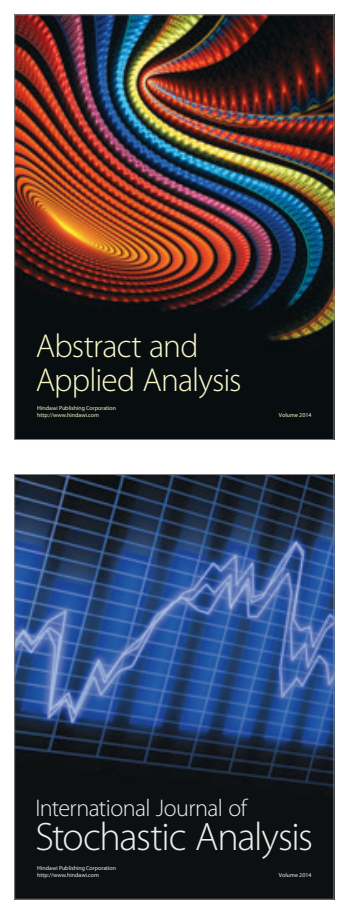

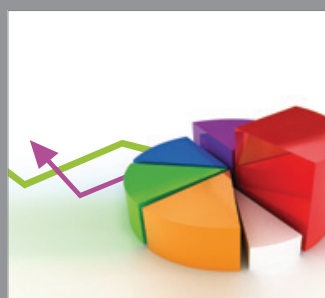

ournal of

Probability and Statistics

Promensencen
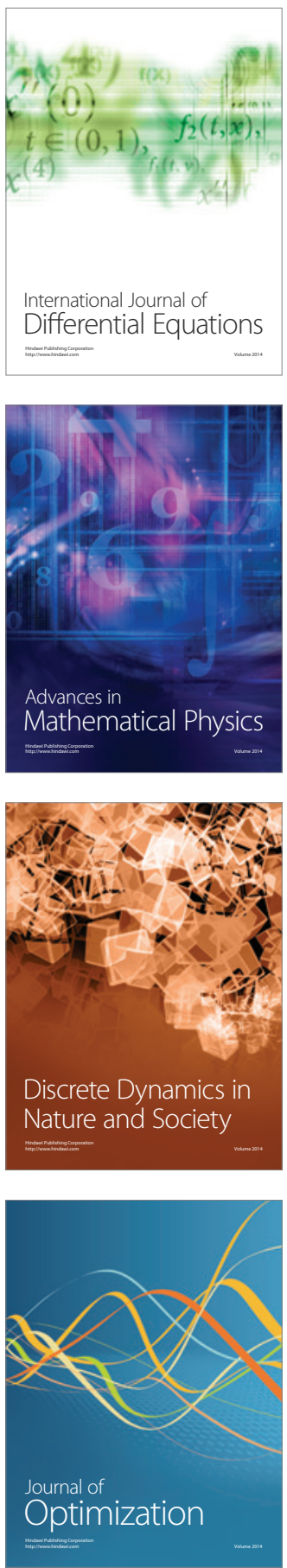